PROCEEDINGS OF THE

AMERICAN MATHEMATICAL SOCIETY

Volume 127, Number 7, Pages 2091-2098

S 0002-9939(99)04890-X

Article electronically published on March 16, 1999

\title{
PROLONGEMENT DE COURANTS POSITIFS A TRAVERS DE PETITS OBSTACLES
}

\author{
JEAN-BAPTISTE POLY ET GILLES RABY
}

(Communicated by Steven R. Bell)

\begin{abstract}
In this paper, we prove an extension theorem through closed subsets having small Haussdorff dimension, for positive currents whose boundary satisfies some growth condition. As a corollary, we get the classical Harvey's extension theorem for closed positive currents. Furthermore, we apply our result to study the boundary of holomorphic chains.
\end{abstract}

\section{INTRODUCTION}

Ce travail a pour objectif essentiel de prouver le

1.1. Théorème. Soit $A$ une partie fermée d'un ouvert $X$ de $\mathbb{C}^{n}$ et $\mathcal{T}$ un courant positif de bidimension $(p, p)$ dans l'ouvert $X \backslash A$. On suppose

(i) La mesure de Haussdorff $\mathcal{H}_{2 p-1}(A)$ est nulle.

(ii) Le courant $b \mathcal{T}$ se prolonge en un courant de masse localement finie dans $X$. Alors, $\mathcal{T}$ se prolonge également en un courant de masse localement finie dans $X$

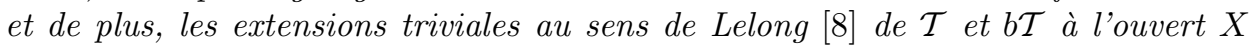
satisfont à l'égalité

$$
b \tilde{\mathcal{T}}=\widetilde{b \mathcal{T}} .
$$

1.2. Remarque. Si $\mathcal{T}$ est fermé dans $X \backslash A$, alors l'hypothèse (ii) est trivialement satisfaite et l'on retrouve ainsi le théorème de prolongement des courants positifs fermés de Harvey [3].

1.3. La section 2 est consacrée à la démonstration du théorème 1.1. Dans la section 3 , nous étudions les courants qui peuvent s'écrire $\mathcal{T}=\mathcal{T}_{1}-\mathcal{T}_{2}$ où $\mathcal{T}_{1}$ et $\mathcal{T}_{2}$ sont des courants positifs de bidimension $(p, p)$ dans l'ouvert $X \backslash A$. Nous montrons par un contre-exemple que le théorème 1.1 ne s'étend pas immédiatement à de tels courants (la condition (i) étant satisfaite, la condition (ii) n'est pas suffisante pour assurer le prolongement souhaité de $\mathcal{T}$ ). Toutefois, nous montrons dans la section 4 comment les considérations de 3 peuvent être exploitées dans le cas où $\mathcal{T}$ est une $p$-chaîne holomorphe dans $X \backslash A$ et plus précisément dans l'étude du problème du bord des chaînes holomorphes au sens de Harvey-Lawson [5, 6], voir aussi [4].

Received by the editors October 14, 1997.

1991 Mathematics Subject Classification. Primary 32C30, 32D15, 53C65, 32C25.

Key words and phrases. Courants positifs, prolongement, mesure de Haussdorff, chaînes holomorphes.

(C)1999 American Mathematical Society 
Nous remercions H. El Mir et N. Sibony pour d'utiles conversations concernant cet article.

\section{Demonstration du theoreme 1.1}

2.1. Nous renvoyons au traité de Federer [2] pour toutes les notions relevant de la théorie géométrique de la mesure. Rappelons maintenant quelques définitions et propriétés usuelles concernant les courants positifs.

Soit $X$ un ouvert de $\mathbb{C}^{n}$ et $\mathcal{D}^{m}(X)$, resp. $\mathcal{D}^{p, q}(X)$, l'espace des formes différentielles $C^{\infty}$ à support compact dans $X$ qui sont de degré $m$, resp. de bidegré $(p, q)$. Un courant $\mathcal{T}$ de bidimension $(p, p)$ dans $X$ est dit positif si pour toutes formes $\alpha_{1}, \ldots, \alpha_{p}$ dans l'espace $\mathcal{D}^{1,0}(X)$, la distribution

$$
\mathcal{T} \wedge i \alpha_{1} \wedge \overline{\alpha_{1}} \wedge \ldots \wedge i \alpha_{p} \wedge \overline{\alpha_{p}}
$$

est une mesure positive sur $X$.

On en déduit que le courant $\mathcal{T}$ est localement de masse finie dans $X$ et on montre que la masse de $\mathcal{T}$ est contrôlée par sa masse de Kähler. De façon plus précise, soit $\Omega=(i / 2) \partial \bar{\partial}\|z\|^{2}$ la forme de Kähler usuelle sur $\mathbb{C}^{n}$ et

$$
\sigma=\mathcal{T} \wedge \frac{\Omega^{p}}{p !}
$$

la mesure trace de $\mathcal{T}$. Il existe une constante $c \geq 1$, ne dépendant que de $n$ et $p$, telle que, pour chaque ouvert $V$ de $X$, on a (voir par exemple Harvey, [4], lemme $1.26)$

$$
\sigma(V) \leq M_{V}(\mathcal{T}) \leq c \sigma(V)
$$

où, lorsque $\Theta$ est un courant de dimension $m$ dans $X, M_{V}(\Theta)$ désigne la masse (finie ou non) de $\Theta$ dans l'ouvert $V$, soit

$$
M_{V}(\Theta)=\sup \left\{\Theta(\varphi) \mid \varphi \in \mathcal{D}^{m}(V) \text { et }|\varphi| \leq 1\right\} .
$$

Cela rappelé, nous donnons maintenant une preuve du théorème 1.

2.2. La relation $b \tilde{\mathcal{T}}=\widetilde{b \mathcal{T}}$ est une simple conséquence de l'existence des extensions. En effet, comme $\mathcal{T}$ et $b \mathcal{T}$ sont des courants localement normaux dans $X \backslash A$, les extensions triviales $\tilde{\mathcal{T}}$ et $\widetilde{b \mathcal{T}}$ sont localement plates dans $X$ de sorte que la différence $b \tilde{\mathcal{T}}-\widetilde{b \mathcal{T}}$ est un courant localement plat de dimension $2 p-1$ à support dans $A$. Comme $\mathcal{H}_{2 p-1}(A)=0$, on a bien l'égalité $b \tilde{\mathcal{T}}-\widetilde{b \mathcal{T}}=0$ d'après le measure support theorem de Federer [2].

2.3. Premier pas. Réduction au cas où $p=n$. Il suffit de recopier la preuve de Harvey [3].

On peut supposer que $X$ est un voisinage de 0 dans $\mathbb{C}^{n}$ et il suffit de montrer que quitte à rétrécir $X$ la masse de $\mathcal{T}$ est bornée dans $X \backslash A$, et pour cela que la masse de Kähler $\sigma(X \backslash A)$ est finie.

Comme $\mathcal{H}_{2 p-1}(A)=0$, a fortiori $\mathcal{H}_{2 p}(A)=0$ et donc, d'après le lemme 2 de Shiffman [10], il existe une base orthonormée de $\mathbb{C}^{n}$ telle que, pour chaque projection $\pi: \mathbb{C}^{n} \rightarrow \mathbb{C}^{p}$ définie par $\pi(z)=\left(z_{k(1)}, \ldots, z_{k(p)}\right)$ où $k$ est l'une des injections croissantes de $\{1,2, \ldots, p\}$ dans $\{1,2, \ldots, n\}$, la fibre $\pi^{-1}\{0\} \cap A$ est réduite au singleton $\{0\}$. Nous désignerons par $\Pi$ l'ensemble des projections $\pi$ qu'on vient de définir. 
Pour une telle projection $\pi$, l'ouvert $X$ contient un polydisque $\Delta=\Delta^{\prime} \times \Delta^{\prime \prime} \subset$ $\mathbb{C}^{p} \times \mathbb{C}^{n-p}$ tel que

- $\Delta$ est relativement compact dans $X$, de sorte que $b \mathcal{T}$ est de masse finie dans $\Delta \backslash A$.

- $A \cap \bar{\Delta}$ ne rencontre pas $\overline{\Delta^{\prime}} \times b \Delta^{\prime \prime}$.

On écrit désormais $\pi$ pour $\left.\pi\right|_{\Delta}$ et on veut montrer que la mesure positive $\mathcal{T} \wedge \pi^{*} \omega_{p}$ est de masse finie dans $\Delta \backslash A$ où $\omega_{p}$ est la forme volume standard dans $\mathbb{C}^{p}$, c'est-àdire

$$
\omega_{p}=\frac{1}{p !}\left((i / 2) \partial \bar{\partial}\|z\|^{2}\right)^{p} .
$$

Ce qui permettra de conclure puisque la mesure trace vérifie

$$
\sigma=\sum_{\pi \in \Pi} \mathcal{T} \wedge \pi^{*} \omega_{p}
$$

Montrons d'abord que la mesure $\mathcal{T} \wedge \pi^{*} \omega_{p}$ ne charge pas $\pi^{-1} \pi A \backslash A$, autrement dit qu'elle est l'extension triviale de sa restriction à $\Delta \backslash \pi^{-1} \pi A$. Pour cela, si $\theta$ est une fonction test positive dans $\Delta \backslash A$, comme $\mathcal{T} \wedge \pi^{*} \omega_{p}$ est un courant localement normal dans $\Delta \backslash A$, on a en utilisant la théorie du slicing, cf. Federer [2]

$$
\left\langle\mathcal{T} \wedge \pi^{*} \omega_{p}, \theta\right\rangle=\int_{z^{\prime} \in \Delta^{\prime}}\left\langle\mathcal{T}, \pi, z^{\prime}\right\rangle(\theta) \omega_{p}\left(z^{\prime}\right)
$$

mais comme la fonction $z^{\prime} \mapsto\left\langle\mathcal{T}, \pi, z^{\prime}\right\rangle(\theta)$ est intégrable et que $\pi A$ est négligeable, il suffit de calculer l'intégrale sur $\Delta^{\prime} \backslash \pi A$.

Quitte à remplacer $\mathcal{T}$ par le produit $\phi \mathcal{T}$ où $\phi: \mathbb{C}^{n} \rightarrow \mathbb{R}^{+}$vaut 1 au voisinage de $A \cap \bar{\Delta}$ et vaut 0 près de $\overline{\Delta^{\prime}} \times b \Delta^{\prime \prime}$, on peut supposer que la projection $\pi$ : supp $\mathcal{T} \backslash \pi^{-1} \pi A \rightarrow \Delta^{\prime} \backslash \pi A$ est propre et on désigne par $\mathcal{T}_{\pi}$ le courant défini dans $\Delta^{\prime} \backslash \pi A$ comme image directe de la restriction de $\mathcal{T}$ à $\Delta \backslash \pi^{-1} \pi A$. Par hypothèse, $b \mathcal{T}_{\pi}$ est de masse finie et il reste à montrer qu'il en est de même de $\mathcal{T}_{\pi}$. Dans l'ouvert $\Delta^{\prime} \backslash \pi A$, le courant $\mathcal{T}_{\pi}$ est défini par une fonction localement intégrable et il suffit d'utiliser dans $\mathbb{C}^{n}=\mathbb{R}^{2 n}$ la variante suivante du théorème de Fubini :

2.4. Deuxième pas. Soit $S$ une partie fermée d'un ouvert $X$ de $\mathbb{R}^{N}$ telle que $\mathcal{H}_{N-1}(S)=0$ et $\mathcal{T}$ le courant défini dans l'ouvert complémentaire $U=X \backslash S$

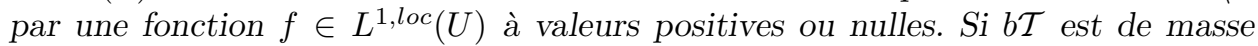
localement finie près de $S$, alors $f$ se prolonge en une fonction localement intégrable dans $X$, autrement dit $\mathcal{T}$ est de masse localement finie près de $S$.

Là encore, le problème est local au voisinage d'un point, disons $0 \in S$. En utilisant la technique précédente, mais ici pour une direction réelle seulement, on peut se ramener au cas où $X=X^{\prime} \times X^{\prime \prime} \subset \mathbb{R}^{N-1} \times \mathbb{R}$, la projection $p: S \rightarrow X^{\prime}$ définie par $p\left(x^{\prime}, x^{\prime \prime}\right)=x^{\prime}$ est propre et de plus, il existe un segment $[-a, a] \subset X^{\prime \prime}$ de sorte que $\operatorname{supp} \mathcal{T}$ est inclus dans $X^{\prime} \times[-a, a]$.

Pour presque tout $x^{\prime} \in X^{\prime}$, on sait que $x^{\prime}$ n'appartient pas à $p S$ et qu'existe la tranche $\left\langle\mathcal{T}, p, x^{\prime}\right\rangle$ avec

$$
\int f\left(x^{\prime}, x^{\prime \prime}\right) d x^{\prime \prime}=\left\langle\mathcal{T}, p, x^{\prime}\right\rangle\left(d x^{\prime \prime}\right)=\left\langle b \mathcal{T}, p, x^{\prime}\right\rangle\left(x^{\prime \prime}\right)
$$

d'où l'on déduit l'inégalité

$$
\int f\left(x^{\prime}, x^{\prime \prime}\right) d x^{\prime \prime} \leq a M_{X}\left(\left\langle b \mathcal{T}, p, x^{\prime}\right\rangle\right)
$$


puis les majorations successives de l'intégrale répétée

$$
\begin{aligned}
\int d x^{\prime} \int f\left(x^{\prime}, x^{\prime \prime}\right) d x^{\prime \prime} & \leq a \int_{x^{\prime} \in X^{\prime} \backslash p S} M_{X}\left(\left\langle b \mathcal{T}, p, x^{\prime}\right\rangle\right) d x^{\prime} \\
& \leq a M_{X \backslash p^{-1} p S}\left(b \mathcal{T} \wedge d x^{\prime}\right) \\
& <\infty
\end{aligned}
$$

ce qui permet de conclure grâce au théorème de Fubini.

\section{ETUde DES DIFFÉRENCES DE COURANTS POSITIFS}

Comme on l'a constaté ci-dessus, la démonstration qui précède se scinde en deux parties où seule la première utilise quelques résultats d'analyse complexe : la positivité permet de se ramener au cas extrême où $p=n$. La seconde est un exercice d'analyse réelle. Afin de préciser ce dernier point, nous rappelons la définition suivante, cf. Burago-Maz'ja ([9], 6.1.1) :

3.1. Définition. Soit $U$ un ouvert de $\mathbb{R}^{N}$. On désigne par $B V(U)$ l'espace des courants réels $\mathcal{T}$ de dimension maximum $N$ dont le bord $b \mathcal{T}$ est globalement de masse finie dans l'ouvert $U$.

Il en résulte immédiatement qu'un tel courant $\mathcal{T}$ est défini par une fonction $f$ localement intégrable dans $U$. Par abus d'écriture, nous désignerons simplement par $f$ le courant défini par la fonction $f$. Pour ces fonctions à variation bornée, les petits obstacles sont inessentiels. De façon précise, on a la

3.2. Proposition. Dans l'ouvert $U$ de $\mathbb{R}^{N}$, soit $A$ une partie fermée dont la mesure de Haussdorff $\mathcal{H}_{N-1}(A)$ est nulle. Alors l'application naturelle de restriction

$$
\rho: B V(U) \rightarrow B V(U \backslash A)
$$

est un isomorphisme.

La preuve de cette proposition s'appuie essentiellement sur le

3.3. Lemme. Sont équivalentes les assertions suivantes, cf. Burago-Maz'ja ([9], 6.1.2) :

- $f \in B V(U)$,

- $|f| \in B V(U)$,

- $f^{+} \& f^{-} \in B V(U)$

et dans ce cas, on a les propriétés suivantes concernant la masse des bords

$$
M_{U}(b f)=M_{U}(b|f|)=M_{U}\left(b f^{+}\right)+M_{U}\left(b f^{-}\right) .
$$

Dans cet énoncé, apparaissent les fonctions positives $f^{+}$et $f^{-}$définies classiquement par les deux relations $f=f^{+}-f^{-}$et $|f|=f^{+}+f^{-}$.

3.4. Preuve de la proposition 3.2. A étant négligeable, l'application $\rho$ est injective. Il reste à étudier la surjectivité. Le lemme 3.3 permet de se ramener au cas des fonctions à variation bornée positives, mais alors la surjectivité de la restriction $\rho$ est un cas particulier du deuxième pas de la section 2. De façon plus précise, soit $\mathcal{T} \in B V(U \backslash A)$ le courant de $U \backslash A$ défini par une fonction $f \in L^{1, l o c}(U \backslash A)$ à valeurs positives ou nulles. D'après le deuxième pas, $\mathcal{T}$ est de masse localement finie près de $A$, donc possède une extension triviale $\tilde{\mathcal{T}}$ à l'ouvert $U$. Comme $\mathcal{H}_{N-1}(A)=0$, d'après le measure support theorem, on obtient l'égalité $b \tilde{\mathcal{T}}=\widetilde{b \mathcal{T}}$ et 
comme l'extension triviale n'augmente pas la masse, l'extension $\tilde{\mathcal{T}}$ de $\mathcal{T}$ appartient bien à $B V(U)$.

3.5. Définition. Soit désormais $X$ un ouvert de $\mathbb{C}^{n}$ et $B V_{p, p}(X)$ l'espace vectoriel réel constitué des courants du type $\mathcal{T}=\mathcal{T}_{1}-\mathcal{T}_{2}$ où $\mathcal{T}_{1}$ et $\mathcal{T}_{2}$ sont des courants positifs de bidimension $(p, p)$ tels que la somme des masses des bords $M_{X}\left(b \mathcal{T}_{1}\right)+M_{X}\left(b \mathcal{T}_{2}\right)$ est finie.

En appliquant le théorème 1.1, on obtient immédiatement le

3.6. Corollaire du théorème 1.1. Soit $A$ une partie fermée de $X$ dont la mesure de Haussdorff $\mathcal{H}_{2 p-1}(A)$ est nulle. Alors, l'application naturelle de restriction

$$
B V_{p, p}(X) \rightarrow B V_{p, p}(X \backslash A)
$$

est un isomorphisme.

3.7. L'exemple qui suit montre que dans la définition de $B V_{p, p}(X)$, on ne peut se borner à supposer seulement la finitude de la masse $M_{X}(b \mathcal{T})$, sauf bien sûr dans le cas extrême où $p=n$, car d'après le lemme $3.3, B V_{n, n}(X)=B V(X)$.

Dans $X=\mathbb{C}^{n} \backslash\{0\}$ avec $n \geq 2$, soit

$$
\mathcal{T}=d\left(\varphi\left(\|z\|^{2}\right) d^{c}\|z\|^{2}\right)
$$

où $\varphi(t)=1 / t^{n+1}$ et $d^{c}=(i / 2)(\bar{\partial}-\partial)$.

$\mathcal{T}$ est un courant fermé, différence de deux courants positifs dans $X$

$$
\mathcal{T}=\varphi\left(\|z\|^{2}\right) d d^{c}\|z\|^{2}+\varphi^{\prime}\left(\|z\|^{2}\right) d\|z\|^{2} \wedge d^{c}\|z\|^{2} .
$$

Cependant, quel que soit $U$ voisinage ouvert de 0 , la restriction de $\mathcal{T}$ à $U \backslash\{0\}$ n'appartient pas à $B V_{n-1, n-1}(U \backslash\{0\})$, car sinon, d'après le corollaire 3.6, le courant $\left.\mathcal{T}\right|_{U \backslash\{0\}}$ serait de masse localement finie au voisinage de 0 , ce qui est inexact.

\section{Chaines holomorphes et Probleme DU Bord}

Cette section 4 est pour l'essentiel consacrée à l'étude du comportement d'un $p$ cycle holomorphe près de certains obstacles de dimension $2 p-1$ dont nous donnons ci-dessous une définition précise.

4.1. Définition. Soit $M$ une partie compacte de $\mathbb{C}^{n}$. On dit que $M$ est une sousvariété $C^{1}$ de dimension $q$ à singularités négligeables si la mesure de Haussdorff $\mathcal{H}_{q}(M)$ est finie et s'il existe un compact $S$ inclus dans $M$ avec $\mathcal{H}_{q}(S)=0$ tel que $M \backslash S$ soit une sous-variété de classe $C^{1}$ fermée dans $\mathbb{C}^{n} \backslash S$, orientable et de dimension $q$.

4.2. Hypothèses. Soit $M$ une partie compacte de $\mathbb{C}^{n}$ qui soit une sous-variété $C^{1}$ de dimension $2 p-1$ à singularités négligeables, $S$ le lieu singulier de $M$ et $\mathcal{Y}$ un $p$ cycle holomorphe dans l'ouvert $\mathbb{C}^{n} \backslash M$ dont le support $Y$ soit relativement compact dans $\mathbb{C}^{n}$.

Nous appelons ici $p$-cycle holomorphe de $X$ tout courant $\mathcal{Y}$ du type

$$
\mathcal{Y}=\sum m_{j}\left[Y_{j}\right]
$$

où $\left(Y_{j}\right)_{j \in J}$ est une famille localement finie d'ensembles analytiques complexes fermés dans $X$ de dimension complexe pure $p$ et chaque $m_{j}$ est un entier, $\left[Y_{j}\right]$ désignant le courant de $X$ défini par intégration sur la sous-variété reg $Y_{j}$ des points réguliers de $Y_{j}$, cf. par exemple Lelong [8]. 
4.3. D'après le théorème 4.7 de Harvey-Lawson [5] qu'on applique dans l'ouvert $V=\mathbb{C}^{n} \backslash S$, le cycle holomorphe $\mathcal{Y}$ est de masse localement finie près de la sousvariété $M \backslash S$, donc possède une extension triviale $\mathcal{T}$ à l'ouvert $V$ laquelle satisfait à la relation

$$
b \mathcal{T}=\sum \lambda_{k}\left[L_{k}\right]
$$

où $\left(L_{k}\right)$ est la famille au plus dénombrable des composantes connexes de la sousvariété $M \backslash S$ et où, pour chaque $k,\left[L_{k}\right]$ désigne le courant d'intégration sur la composante $L_{k}$ où l'on a choisi une orientation et $\lambda_{k}$ est un entier.

On peut aisément construire des exemples où le courant $\mathcal{T}$ n'est pas de masse finie dans l'ouvert $V$ : il suffit d'empiler dans $\mathbb{C} \backslash\{0\}$ des disques troués à l'origine. Toutefois, on a

4.4. Théorème. Avec les hypothèses 4.2 et les notations qui précèdent, si le courant $b \mathcal{T}$ est de masse finie dans $V$, alors il en est de même du courant $\mathcal{T}$. De plus si $\mathcal{Z}$ et $\mathcal{C}$ désignent respectivement les extensions triviales de $b \mathcal{T}$ et $\mathcal{T} a ̀ \mathbb{C}^{n}$, alors $\mathcal{Z}$ est un cycle rectifiable qui borde la p-chaîne holomorphe $\mathcal{C}$.

Précisons d'abord ce que nous entendons ici par chaîne holomorphe dans un ouvert $X$ de $\mathbb{C}^{n}$.

4.5. Définition. Nous appelons $p$-chaîne holomorphe de $X$ tout courant $\mathcal{C}$ de masse localement finie dans $X$ qui de plus satisfait les conditions suivantes :

- Si $Z$ désigne le support du bord $b \mathcal{C}$, la restriction de $\mathcal{C}$ à l'ouvert $X \backslash Z$ est un $p$-cycle holomorphe.

- $\mathcal{C}$ est l'extension triviale de sa restriction à l'ouvert $X \backslash Z$.

Ainsi, un $p$-cycle holomorphe n'est autre qu'une $p$-chaîne holomorphe fermée. On aura noté que dans toute cette section 4 , cycle est mis pour courant fermé. Ceci explique pourquoi nous avons choisi d'appeler cycles holomorphes de $X$ les courants du type $(*)$, suivant en cela la terminologie de King [7], réservant le terme chaînes holomorphes de $X$ pour les courants $\mathcal{C}$ de la définition 4.5.

Notre définition d'une chaîne holomorphe est peut-être trop grossière, mais suffira pour les besoins de cet article.

4.6. Démonstration du théorème 4.4. Comme dans 2.2, la dernière partie de ce théorème est conséquence immédiate de l'existence des extensions triviales $\mathcal{Z}$ et $\mathcal{C}$. En effet, le courant $\mathcal{Z}-b \mathcal{C}$ est alors localement plat de dimension $2 p-1$ et à support dans $S$. Comme $\mathcal{H}_{2 p-1}(S)=0$, on a bien $\mathcal{Z}-b \mathcal{C}=0$ d'après le measure support theorem de Federer.

Il reste donc à montrer que $\mathcal{T}$ est de masse finie. Soit

$$
\mathcal{Y}=\mathcal{Y}^{+}-\mathcal{Y}^{-}
$$

l'écriture canonique du cycle holomorphe $\mathcal{Y}$ comme différence de deux cycles holomorphes positifs. Comme $\mathcal{Y}$, ces deux cycles ont des extensions triviales $\mathcal{T}^{+}$et $\mathcal{T}^{-}$ à l'ouvert $V$. Cela dit, si les supports de $b \mathcal{T}^{+}$et $b \mathcal{T}^{-}$ne sont pas nécessairement disjoints (comme c'était le cas dans Harvey [4], page 367), l'étude locale le long de la sous-variété $M \backslash S$ qu'on détaille dans 4.7 montre que

$$
M_{V}(b \mathcal{T})=M_{V}\left(b \mathcal{T}^{+}\right)+M_{V}\left(b \mathcal{T}^{-}\right)
$$

Mais alors $\mathcal{T}=\mathcal{T}^{+}{ }_{-} \mathcal{T}^{-}$appartient à $B V_{p, p}(V)$, ce qui permettra de conclure grâce au corollaire 3.6. 
4.7. Rappelons le début du lemme 3.24 de Harvey [4].

Il existe un compact $A$ de $M$ avec $\mathcal{H}_{2 p-1}(A)=0$ tel que tout point $\xi$ de $M \backslash A$ possède un voisinage $B$ avec les propriétés suivantes. L'ensemble analytique $W=$ $Y \cap B$ est une sous-variété de $B \backslash M$ et on a l'alternative suivante

(i) $W$ est connexe et le couple $(W, M \cap B)$ est une sous-variété $C^{1}$ à bord.

(ii) $\bar{W}=W \cup(M \cap B)$ est une sous-variété analytique complexe connexe dans laquelle $M \cap B$ est une hypersurface de classe $C^{1}$ divisant $W$ en deux composantes $W_{1}$ et $W_{2}$.

La preuve donnée par Harvey utilise la construction explicite de $\mathcal{Y}$ comme solution d'un problème de bord. Cependant, les propriétés de régularité locale sont valides pour un cycle holomorphe quelconque, cf. Chirka [1] (19.1, Regularity near the boundary).

Tout comme $\mathcal{T}$, les courants $\mathcal{T}^{+}$et $\mathcal{T}^{-}$satisfont aux relations

$$
b \mathcal{T}^{+}=\sum \lambda_{k}^{+}\left[L_{k}\right] \quad \text { et } \quad b \mathcal{T}^{-}=\sum \lambda_{k}^{-}\left[L_{k}\right] .
$$

Pour montrer que $M_{V}(b \mathcal{T})=M_{V}\left(b \mathcal{T}^{+}\right)+M_{V}\left(b \mathcal{T}^{-}\right)$, il suffit de montrer que pour chaque $k$, on a $\left|\lambda_{k}\right|=\left|\lambda_{k}^{+}\right|+\left|\lambda_{k}^{-}\right|$. C'est donc un problème local qu'il suffit d'étudier au voisinage d'un point de régularité. Dans le voisinage $B$ d'un point $\xi$ de type (ii), on peut écrire $\mathcal{T}=\lambda_{1}\left[W_{1}\right]+\lambda_{2}\left[W_{2}\right]$ où $\lambda_{1}$ et $\lambda_{2}$ sont des entiers. Il reste seulement à examiner le cas où $\lambda_{1} \lambda_{2}<0$, puisque dans les autres cas (y compris les cas de type (i)), $\mathcal{T}$ coïncide dans $B$ soit avec $\mathcal{T}^{+}$, soit avec $\mathcal{T}^{-}$. On restreint désormais la situation à l'ouvert $B$. En supposant par exemple $\lambda_{1}>0>\lambda_{2}$, on a $\mathcal{T}^{+}=\lambda_{1}\left[W_{1}\right]$ et $\mathcal{T}^{-}=-\lambda_{2}\left[W_{2}\right]$. Si l'orientation de $L=M \cap B$ est telle que $b\left[W_{1}\right]=[L]$, on obtient $b \mathcal{T}^{+}=\lambda_{1}[L]$ tandis que $b \mathcal{T}^{-}=\lambda_{2}[L]$, de sorte qu'on a bien

$$
M_{B}\left(b \mathcal{T}^{+}\right)+M_{B}\left(b \mathcal{T}^{-}\right)=\left(\lambda_{1}-\lambda_{2}\right) M_{B}[L]=M_{B}(b \mathcal{T})
$$

puisque $b \mathcal{T}=\left(\lambda_{1}-\lambda_{2}\right)[L]$.

4.8. Application au problème du bord. Soit $X$ un ouvert de $\mathbb{C}^{n}$ et $\mathcal{Z}$ un cycle localement rectifiable de dimension $2 p-1$ dans $X$. Résoudre le problème du bord, cf. $[4,5,6]$, c'est chercher des conditions nécessaires et suffisantes pour que $\mathcal{Z}$ borde une $p$-chaîne holomorphe $\mathcal{C}$ de $X$. Par abus de langage, nous dirons que le $p$-cycle holomorphe $\mathcal{Y}$, restriction de $\mathcal{C}$ à l'ouvert $X \backslash \operatorname{supp} \mathcal{Z}$, est solution du problème du bord dans $X$ pour la donnée $\mathcal{Z}$. Cela dit, il résulte immédiatement du théorème 4.4 la

4.9. Proposition. Soit $\mathcal{Z}$ un cycle rectifiable de dimension $2 p-1$ dans $\mathbb{C}^{n}$ dont le support est une sous-variété $C^{1}$ à singularités négligeables, $S$ le lieu singulier de $M$. Si $\mathcal{Y}$ est un $p$-cycle holomorphe dans $\mathbb{C}^{n} \backslash M$, solution du problème du bord dans l'ouvert $\mathbb{C}^{n} \backslash S$ pour la donnée $\left.\mathcal{Z}\right|_{\mathbb{C}^{n} \backslash S}$, alors $\mathcal{Y}$ est solution du même problème dans $\mathbb{C}^{n}$ pour la donnée $\mathcal{Z}$.

\section{REFERENCES}

1. E.M. Chirka, Complex analytic sets, Kluwer Academic Pub., Dordrecht, 1989. MR 92b:32016

2. H. Federer, Geometric measure theory, Springer-Verlag, New-York, 1969. MR 41:1976

3. R. Harvey, Removable singularities for positive currents, Amer. J. Math. 96 (1974), 67-78. MR 50:13602

4. R. Harvey, Holomorphic chains and their boundaries, Proc. Symp. Pure Math. 30 Part 1 (1977), 309-382. MR 56:5929

5. R. Harvey and B. Lawson, On boundaries of complex analytic varieties I, Ann. of Math. 102 (1975), 233-290. MR 54:13130 
6. R. Harvey and B. Lawson, On boundaries of complex analytic varieties II, Ann. of Math. 106 (1977), 213-238. MR 58:17186

7. J. King, The currents defined by analytic varieties, Acta Math. 127 (1971), 185-220. MR 52:14359

8. P. Lelong, Intégration sur un ensemble analytique complexe, Bull. Soc. Math. France $\mathbf{8 5}$ (1957), 239-262. MR 20:2465

9. V. Maz'ja, Sobolev spaces, Springer-Verlag, Berlin, 1985. MR 87g:46056

10. B. Shiffman, On the removal of singularities of analytic sets, Michigan Math. J. 15 (1968), 111-120. MR 37:464

ESA CNRS 6086 Groupes de Lie et Géométrie, Laboratoire de Mathematiques, Universite de Poitiers, 40 Avenue du Recteur Pineau, 86022 Poitiers, France

E-mail address: poly@wallis.univ-poitiers.fr

E-mail address: raby@mathrs.univ-poitiers.fr 